\title{
EL IMPACTO INSTITUCIONAL EN EL EMPRENDIMIENTO DE MÉXICO
}

\section{INSTITUTIONAL IMPACT ON MEXICAN ENTREPRENEURSHIP}

Osorio Novela, Germán (Universidad Autónoma de Baja California, México) *

Saavedra Leyva, Rafael Eduardo (Universidad Autónoma de Baja California, México) **

Martínez Sidón, Gilberto (Instituto Nacional de la Paz, Baja California Sur, México) ${ }^{* * *}$

\section{Resumen:}

El dinamismo del emprendimiento representa quizá el elemento más básico para las economías de mercado, ofreciendo distintos beneficios productivos y sociales, cuyo alcance depende de las condiciones en cómo éste se genere. Por ello, esta investigación analiza la relación entre la actividad emprendedora y algunas de las conductas públicas de México, en donde el acceso y apertura gubernamental, la percepción de la inseguridad y la calidad de los servicios públicos representan las variables institucionales de este estudio. A través del método de corte transversal, los hallazgos sugieren que la calidad de los servicios públicos, el acceso y apertura gubernamental son elementos institucionales significativos al momento de iniciar un negocio en diversas regiones de México, mientras que la percepción de la inseguridad no resulta ser un factor estadísticamente significativo, lo que ofrece pautas para su pertinente análisis.

Palabras claves: Emprendimiento, Instituciones, Eficiencia gubernamental.

JEL: L26, M13, D21, E02.

\begin{abstract}
The dynamism of entrepreneurship represents perhaps the most basic element for market economies, offering different productive and social benefits, whose scope depends on the conditions in how it is generated. Therefore, this research analyzes the relationship between entrepreneurial activity and some of the public behaviors in Mexico, where government access and openness, the perception of insecurity and the quality of public services represent the institutional variables of this study. Through the cross-sectional method, the findings suggest that the quality of public services, government access and openness are significant institutional elements at the time of starting a business, while the perception of insecurity is not a statistically significant factor, what offers guidelines for its pertinent analysis.
\end{abstract}

JEL: L26, M13, D21, E02.

Key words: Entrepreneurship; Institutions; Government efficiency.

* Universidad Autónoma de Baja California, gosorio@uabc.edu.mx.

** Universidad Autónoma de Baja California, rsaavedra@uabc.edu.mx.

*** gilberto.mtzs88@gmail.com

Recibido: 29 de diciembre de 2018. Aceptado: 16 de mayo de 2019. 


\section{INTRODUCCIÓN}

En economías de mercado, se reconoce al emprendimiento como un factor que ayuda a elevar el desarrollo económico, a través de la creación de empleo, nuevas fuentes de ingreso y distribución de riqueza. En México, el gobierno, como ente del sistema económico, tiene la misión de proveer una estructura con instituciones públicas sólidas, capaces de potenciar las actividades productivas de las diversas regiones. Por ello, surge la necesidad de analizar cómo las instituciones impactan sobre el emprendimiento.

Uno de los principales objetivos de las instituciones gubernamentales es brindar servicios de calidad, que satisfagan las necesidades de la sociedad, pues si éstos no cumplen con lo que demandan las personas, la percepción sobre la eficiencia de las instituciones será negativa (Chingos, 2012). En contra parte, sirvaN de ejemplo diversos estudios empíricos que señalan que el correcto funcionamiento de las instituciones, a través de los servicios públicos que se brindan, genera una imagen positiva de éstas ante la sociedad, coadyuvando en la generación y consolidación de negocios productivos (Urbano y Álvarez, 2014; Nystrom, 2008).

Se ha sostenido desde tiempo atrás que, al elevar la calidad de los servicios públicos, se incrementa el nivel de satisfacción y percepción positiva de los ciudadanos hacia los gobiernos, generando condiciones propicias para incentivar actividades económicas productivas (Swianiewicz, 2001). Sin embargo, en muchos casos el desinterés de las personas para participar sobre temas de política pública, conlleva al desinterés de las instituciones en cumplir con sus objetivos, limitando las actividades económicas en ambientes propicios para su eventual éxito.

El Banco Interamericano de Desarrollo (BID, 2011) señala que, en los países de América Latina, las instituciones cuentan con poca capacidad para dar solución a los problemas que enfrenta la sociedad, lo cual provoca, entre otras cosas, un descontento social generalizado que se traduce en un bajo nivel de confianza hacia las instituciones. Como argumentan Van de Walle y Geert (2003), esta desconfianza se atribuye a un mal funcionamiento de las actividades para las cuales fueron establecidas.

El caso de México no dista de ser diferente, pues como señala Tapia (2010), en el país se ha tenido la constante percepción de que el gobierno es responsable de solventar las necesidades sociales y crear condiciones económicas de desarrollo adecuados para la sociedad. Es por ello que cuando estas necesidades no son cubiertas, la precepción de la sociedad hacia el sector público es que no está realizando su trabajo con eficiencia, afectando actividades productivas y sociales, tal como se plantea en Acs et al. (2018).

Ante un escenario de falta de credibilidad de las personas en el gobierno, éste ha optado en los últimos años, por recurrir al uso de las redes sociales e internet para crear sistemas de interacción entre la sociedad y las instituciones públicas, a través de la creación de nuevas estructuras, como gobierno electrónico, ahora catalogado digital. Al respecto, Tolbert y Mossberger (2006), Belanger y Carter (2008) y Horsburgh et al (2011) sugieren que el gobierno electrónico tiene como objetivo facilitar los trámites gubernamentales, la disposición de la información y atender las demandas de la sociedad. Por consiguiente, este instrumento intenta elevar la confianza y eliminar, en la medida de lo posible, la percepción negativa y deficiencia de las instituciones. Los autores sugieren que las personas que mayormente utilizan esta herramienta, tienden a confiar más en los servicios que proporciona el gobierno.

Con este contexto, el objetivo de este estudio es determinar cómo la eficiencia institucional impacta sobre el nivel de emprendimiento en México; considerando a la actividad emprendedora 
como uno de los eslabones básicos para el crecimiento económico, a través del aumento de la capacidad productiva del país; así como del desarrollo económico, en tanto mecanismo óptimo de mercado para la distribución de riqueza. La hipótesis de esta investigación es que el grado de eficiencia institucional, reflejado en indicadores como apertura gubernamental, percepción de inseguridad y calidad de servicios públicos, condiciona el nivel de emprendimiento de una economía. Específicamente, la pregunta que se quiere responder es ¿cuál es el grado de implicación en la actividad emprendedora ante el contexto actual de la eficiencia institucional en México?

Para realizar este análisis, el documento se estructura de la siguiente manera: además de este apartado introductorio, en la segunda sección se describen los enfoques convencionales y alternos que explican el dinamismo del emprendimiento y el marco institucional; en el tercer apartado se exponen evidencias empíricas contemporáneas en donde se reflejan las distintas relaciones entre variables institucionales y económicas, específicamente asociadas a la apertura empresarial, en contextos regionales diversos; la metodología estadística y econométrica de las variables utilizadas y sus estimaciones se detallan en el apartado cuatro; mientras que en el apartado cinco de resultados se desarrolla un análisis descriptivo sobre la relación existente entre indicadores de emprendimiento y los factores institucionales, complementado con una sección de análisis de los hallazgos econométricos. Finalmente se muestran las conclusiones y las referencias bibliográficas utilizadas.

\section{LA TEORÍA DEL EMPRENDIMIENTO Y SU VINCULACIÓN CON LA TEORÍA INSTITUCIONAL}

El fomento de la empresarialidad, entendida como la capacidad de crear y desarrollar empresas, es un aspecto clave de la política pública en numerosos países, dada la importancia en el crecimiento y desarrollo de mercados y economías. El dinamismo emprendedor está estrechamente vinculado con el descubrimiento y aprovechamiento de oportunidades rentables, las cuales tienen un efecto favorable en los sectores productivos, al ser un proceso de mercado natural para abastecer nuevos productos o renovar bienes y empresas ineficientes por otras más competitivas (Kantis et al, 2004; Shane y Venkataraman, 2000).

En este sentido, el proceso para emprender está dado por una serie de decisiones racionales y secuenciales, ya sea de manera individual o colectiva. Este comienza con el descubrimiento de una oportunidad de mercado. Posteriormente, entra en juego la determinación de su viabilidad, para ello se evalúa el valor esperado del beneficio y el costo, bajo un comportamiento racional. Por último, se toma la decisión de aprovechar la oportunidad a través del emprendimiento de una nueva empresa, o comercializar su idea con otra organización ya existente (Plummer et al, 2007; Kantis, 2008). Principalmente, es en este último paso en donde las decisiones de emprender no sólo se condicionan por características internas a los potenciales emprendedores (ejemplo: habilidades y conocimientos), sino por factores externos, tales como acceso a recursos financieros, no financieros y contextos institucionales.

El marco institucional, es en términos simples como las reglas del juego para la convivencia social y productiva, son determinantes para la consolidación de las oportunidades de mercado, a través del emprendimiento viable, estructurado y exitoso (Luksha, 2008). Un entorno institucional confiable y eficiente proporciona nichos de mercado y la promoción de actividades emprendedoras con los recursos externos necesarios para su sostenibilidad. De esta forma, el círculo institucional es un elemento clave para el análisis del fenómeno del emprendimiento. 
La literatura institucional resalta tres dimensiones políticas que determinan el dinamismo del emprendimiento: la educación y cultura; las acciones de apoyo y la legislación. Las políticas de educación y cultura se orientan en instruir y motivar a las personas a emprender. Las políticas de apoyo consisten en dar soporte a los nuevos negocios, por medio del acceso a recursos financieros y no financieros tales como consultoría técnica, gerencial, crédito y seguimiento. Mientras que las políticas legislativas se presentan con el surgimiento de nuevos estatutos, reglamentos y leyes capaces de respaldar y mejorar el entorno empresarial (Davari y Farokhmanesh, 2017).

Los enfoques teóricos convencionales sostienen que los países se pueden incorporar a la senda del crecimiento económico a medida que éstos incentiven y fortalezcan la actividad emprendedora, específicamente los emprendimientos surgidos en contextos de innovación tecnológica en procesos o productos, también llamados emprendimientos por oportunidad (Acs y Vargas, 2005). Así, se sugiere que la calidad institucional, a través de la actividad emprendedora, tiene un impacto en el crecimiento económico. Se argumenta que el emprendimiento es la conexión entre los factores institucionales y el crecimiento de las economías. Esta tesis se ha contrastado a través de diversos estudios en donde se utilizan variables institucionales tales como el control de la corrupción, acceso a servicios públicos, cobertura privada, densidad empresarial y nuevas empresas (Bosma et al., 2018; Aparicio et al., 2015).

No obstante, desde una visión más amplia, la evidencia empírica ha demostrado la existencia de emprendimientos que obedecen a otros dinamismos diferentes al de la oportunidad, llamados por necesidad, la cual no está vinculada al descubrimiento de nichos de mercado o innovación tecnológica, sino a la imperante necesidad por parte del emprendedor de conseguir ingresos monetarios, en forma de autoempleo. La mayoría de las veces, esta conducta de mercado es ocasionada por el contexto de vulnerabilidad y marginación en el que viven sectores poblacionales en países en vías de desarrollo (Mungaray et al., 2017).

Los emprendimientos por necesidad han sido estudiados en marcos teóricos alternos, comúnmente catalogados con el término de push effect o recession push, ya que las características principales de estos dinamismos de mercado se asocian al surgimiento de nuevas empresas a micro escala (de 1 a 5 trabajadores), principalmente de autoempleo, en donde sólo labora el dueño o empleados provenientes del mismo nicho familiar. En este sentido, factores intangibles como capacitación, consultoría y nivel educativo de los dueños, se vuelven determinantes para el incremento competitivo y permanencia en el mercado de este tipo de empresas (Fairlie y Fossen, 2017; Mungaray et al., 2016). La mayoría de estos factores intangibles están condicionados por la calidad institucional de la región.

En este sentido, el marco institucional es un aspecto de la mayor relevancia para determinar las tendencias en el número y tipo de emprendimientos. La conformación institucional de una economía afecta a la dinámica empresarial. Este entorno se caracteriza por la interacción y el desarrollo de actividades que involucra, entre otros elementos, a la calidad del gobierno, el acceso de capital y a las percepciones de los empresarios, principalmente (Acs et al., 2008). Desde tiempo atrás, diversa literatura ha señalado la existencia de factores institucionales capaces de condicionar la reacción de los empresarios e individuos para emprender nuevos negocios (North, 1993).

Se ha comprobado cómo el ámbito institucional condiciona el grado de crecimiento y eventual desarrollo de las economías, al ser un factor, si bien no suficiente, sí necesario para la eficiencia productiva y de mercado. Algunas teorías institucionales y neoinstitucionales, iniciadas por Schumpeter y Douglass C. North, consideran a este ámbito como determinante principal para explicar el grado de desarrollo de los países. Estos estudios se han multiplicado en los últimos años, 
en donde el ámbito institucional, como variable de análisis, ha tenido una presencia constante en las investigaciones teóricas de la ciencia económica, si bien primero marginal, poco a poco con mayor relevancia e impacto, hasta considerarlo como elemento indispensable para su incorporación en los programas de investigación científica de la economía y sus diversos enfoques. Hoy día, se ha establecido que, en tanto no se considere al contexto institucional en las políticas de desarrollo de los países emergentes, éstas seguirán fracasando irremediablemente (González, 2009).

Dentro de esta línea de investigación se ubica el trabajo de Acs et al. (2018), quienes estudian la relación entre las instituciones, el emprendimiento y crecimiento económico. Su trabajo aboga por otorgar a la iniciativa emprendedora, junto con el ente institucional, un rol clave en crecimiento económico, asumiendo a ambos conceptos como los factores omitidos en la función de producción agregada. Sus resultados sugieren al emprendimiento e instituciones como componentes relevantes para explicar las tasas de crecimiento de las naciones.

Además de estimular crecimiento económico, la iniciativa emprendedora también es generadora de fuentes de trabajo y distribución de riqueza, así lo afirma la investigación de De Wit y De Kok (2013). En donde se analiza, a través del método de clasificación dinámica, cómo las empresas de menor tamaño contribuyen en una mayor proporción a la creación de empleo, en comparación a sus contrapartes más grandes. Adicionalmente, para Amorós y Cristi (2011), el emprendimiento es una herramienta para combatir la pobreza. Los autores exponen que la actividad emprendedora es una pieza importante en las naciones con un menor ingreso per cápita y mayor desigualdad. Sus resultados exhiben que el espíritu emprendedor influye como reductor de pobreza en los países del estudio.

Es quizá el ente gubernamental el actor principal del ámbito institucional con mayor afectación en el sistema económico. El gobierno asume el rol dirigente y rector de la actividad económica, convirtiéndose en factor de estabilidad e impulso del crecimiento y desarrollo. El emprendimiento debe ser la principal herramienta en sistemas de mercado, que enlace ambas dimensiones. El desarrollo y tendencias del emprendimiento es sin duda un reflejo de la convergencia teórica y práctica entre los enfoques económicos e institucionales. Su eficiencia y calidad es entonces determinante para el desarrollo de los países.

\section{EVIDENCIAS EMPÍRICAS DE LAS TENDENCIAS DEL EMPRENDIMIENTO CONDICIONADAS A LA CALIDAD INSTITUCIONAL}

Existen investigaciones empíricas sobre la relación entre emprendimiento e instituciones (cuadro 1). Desai et al. (2003) analizan la influencia de las instituciones en la tasa de entrada, tasa de salida y en el tamaño de las empresas. Sus resultados exhiben un rol importante del entorno institucional en la dinámica de las nuevas empresas, especialmente la protección de los derechos de propiedad que aumentan la entrada de nuevos negocios y disminuyen las tasas de salida. Mientras la legitimidad afecta el tamaño del negocio, permitiendo el desarrollo de empresas pequeñas, en contraste con firmas más grandes. 
Osorio Novela, G., Saavedra Leyva, R. E., Martínez Sidón, G.

\section{CUADRO 1. EVIDENCIA EMPÍRICA SOBRE EMPRENDIMIENTO E INSTITUCIONES}

\begin{tabular}{|c|c|c|c|c|}
\hline Autor(es) & Emprendimiento & Instituciones & Metodología & Hallazgo \\
\hline $\begin{array}{l}\text { Estrin, Aidis } \\
\mathrm{y} \\
\text { Mickiewicz. } \\
(2007)\end{array}$ & $\begin{array}{l}\text { Emprendedores } \\
\text { nacientes, GEM }\end{array}$ & $\begin{array}{l}\text { Percepción de la } \\
\text { corrupción, } \\
\text { Transparency } \\
\text { International } \\
\end{array}$ & $\begin{array}{l}\text { Modelo } \\
\text { probabilístico }\end{array}$ & $\begin{array}{l}\text { El entorno institucional detiene la } \\
\text { actividad emprendedora }\end{array}$ \\
\hline $\begin{array}{l}\text { Nystrom } \\
\text { (2008) }\end{array}$ & $\begin{array}{ll}\text { Tasa } & \text { de } \\
\text { autoempleo, } & \\
\text { COMPEDIA } & \end{array}$ & $\begin{array}{lr}\text { Se emplean } & \text { varios } \\
\text { parámetros } & \text { que } \\
\text { componen el índice } \\
\text { de } & \text { libertad } \\
\text { económica, Fraser } \\
\text { Instituto }\end{array}$ & $\begin{array}{l}\text { Datos de } \\
\text { panel }\end{array}$ & $\begin{array}{llr}\text { Los gobiernos } & \text { con } & \text { mejor } \\
\text { estructura legal y mayor seguridad } \\
\text { aumentan la } & \text { actividad } \\
\text { emprendedora } & & \end{array}$ \\
\hline $\begin{array}{l}\text { Friedman } \\
\text { (2011) }\end{array}$ & $\begin{array}{l}\text { Emprendimiento } \\
\text { por oportunidad, } \\
\text { GEM }\end{array}$ & $\begin{array}{l}\text { Índice de } \\
\text { gobernanza mundial, } \\
\text { World Governence } \\
\text { Indicators }\end{array}$ & $\begin{array}{l}\text { Matriz de } \\
\text { correlación }\end{array}$ & $\begin{array}{l}\text { La relación del emprendimiento y } \\
\text { el índice de gobernanza mundial } \\
\text { es negativa }\end{array}$ \\
\hline $\begin{array}{l}\text { Álvarez y } \\
\text { Urbano } \\
(2012)\end{array}$ & $\begin{array}{l}\text { Tasa de actividad } \\
\text { emprendedora, } \\
\text { GEM }\end{array}$ & $\begin{array}{l}\text { Estabilidad } \\
\text { y colítica } \\
\text { corrupción, } \\
\text { Governence } \\
\text { Indicators }\end{array}$ & $\begin{array}{l}\text { Datos de } \\
\text { panel }\end{array}$ & $\begin{array}{l}\text { Las instituciones informales } \\
\text { afectan en el emprendimiento de } \\
\text { las naciones con ingreso medio y } \\
\text { bajo }\end{array}$ \\
\hline $\begin{array}{l}\text { Méndez, } \\
\text { Galindo y } \\
\text { Ribeiro } \\
(2012) \\
\end{array}$ & $\begin{array}{l}\text { Actividad } \\
\text { emprendedora total, } \\
\text { GEM }\end{array}$ & $\begin{array}{l}\text { Indicadores de } \\
\text { gobernabilidad, } \\
\text { World Governence } \\
\text { Indicators }\end{array}$ & $\begin{array}{l}\text { Mínimo } \\
\text { cuadrados } \\
\text { generalizados }\end{array}$ & $\begin{array}{l}\text { La gobernabilidad aumenta el } \\
\text { emprendimiento, y genera } \\
\text { crecimiento económico }\end{array}$ \\
\hline $\begin{array}{l}\text { Valdez y } \\
\text { Richardson } \\
\text { (2013) }\end{array}$ & $\begin{array}{l}\text { Emprendimiento } \\
\text { por oportunidad y } \\
\text { necesidad, GEM }\end{array}$ & $\begin{array}{l}\text { Dimensiones } \\
\text { institucionales, } \\
\text { diversas fuentes }\end{array}$ & $\begin{array}{l}\text { Mínimos } \\
\text { cuadrados } \\
\text { ordinarios }\end{array}$ & $\begin{array}{l}\text { Las dimensiones institucionales } \\
\text { influyen en la actividad } \\
\text { emprendedora }\end{array}$ \\
\hline $\begin{array}{l}\text { Avnimelech, } \\
\text { Zelekha y } \\
\text { Sharabi } \\
\text { (2014) }\end{array}$ & $\begin{array}{l}\text { Tasa de } \\
\text { emprendimiento, } \\
\text { LinkedIn }\end{array}$ & $\begin{array}{l}\text { Índice } \\
\text { corrupción, } \\
\text { Transparency } \\
\text { International }\end{array}$ & $\begin{array}{l}\text { Mínimos } \\
\text { cuadrados } \\
\text { ordinarios }\end{array}$ & $\begin{array}{l}\text { El efecto de la corrupción al } \\
\text { emprendimiento es menor en los } \\
\text { países en vía de desarrollo que en } \\
\text { las naciones desarrolladas }\end{array}$ \\
\hline $\begin{array}{l}\text { García- } \\
\text { Posada y } \\
\text { Mora- } \\
\text { Sanguinetti } \\
(2014) \\
\end{array}$ & $\begin{array}{l}\text { Tasa de entrada del } \\
\text { autoempleo, } \\
\text { Instituto Nacional } \\
\text { de Estadística de } \\
\text { España } \\
\end{array}$ & $\begin{array}{l}\text { Eficiencia judicial, } \\
\text { Consejo General de } \\
\text { Poder Judicial de } \\
\text { España }\end{array}$ & $\begin{array}{l}\text { Datos de } \\
\text { panel }\end{array}$ & $\begin{array}{l}\text { La tasa de entrada del autoempleo } \\
\text { disminuye por la eficiencia } \\
\text { judicial }\end{array}$ \\
\hline $\begin{array}{l}\text { Urbano y } \\
\text { Álvarez } \\
(2014)\end{array}$ & $\begin{array}{l}\text { Actividad } \\
\text { emprendedora total, } \\
\text { GEM }\end{array}$ & $\begin{array}{l}\text { Dimensiones } \\
\text { institucionales, se } \\
\text { obtuvieron a través } \\
\text { de diversas fuentes } \\
\end{array}$ & $\begin{array}{l}\text { Modelo de } \\
\text { regresión } \\
\text { logística }\end{array}$ & $\begin{array}{l}\text { Las dimensiones institucionales } \\
\text { aumentan la posibilidad de } \\
\text { emprender de los individuos }\end{array}$ \\
\hline $\begin{array}{l}\text { Mohammadi } \\
\text { (2017) }\end{array}$ & $\begin{array}{l}\text { Emprendimiento } \\
\text { productivo, GEM }\end{array}$ & $\begin{array}{l}\text { Corrupción y calidad } \\
\text { institucional, } \\
\text { Transparency } \\
\text { International } \\
\text { World Bank }\end{array}$ & $\begin{array}{l}\text { Análisis de } \\
\text { regresión } \\
\text { múltiple }\end{array}$ & $\begin{array}{l}\text { La corrupción se relaciona } \\
\text { inversamente con el } \\
\text { emprendimiento } \\
\text { mientras la calidad institucional se } \\
\text { relaciona directamente con el } \\
\text { emprendimiento }\end{array}$ \\
\hline $\begin{array}{l}\text { Samadi } \\
\text { (2019) }\end{array}$ & $\begin{array}{l}\text { Emprendimiento } \\
\text { por oportunidad, } \\
\text { GEM }\end{array}$ & $\begin{array}{lr}\text { Derechos } & \text { de } \\
\text { propiedad, } & \\
\text { International } & \\
\text { Property } & \text { Rights } \\
\text { Index } & \\
\end{array}$ & $\begin{array}{l}\text { Causalidad } \\
\text { de Granger }\end{array}$ & $\begin{array}{l}\text { La causalidad es bidireccional en } \\
\text { el largo plazo para los países en } \\
\text { etapa de innovación }\end{array}$ \\
\hline
\end{tabular}

Fuente: Elaboración propia con base en Saavedra y Texis, 2018. 
Para Acs (2006), la decisión de emprender está influenciada por marcos de condiciones empresariales y generales de la nación. Por condiciones empresariales se entiende a aquellas características que influyen en el entorno empresarial como capital de riesgo, financiamiento, capacitación, aspecto social y cultural. Mientras las condiciones generales de la nación se definen como la capacidad del país por fomentar la creación de empresas. Dentro de este marco de condiciones se destaca la gobernabilidad, apertura comercial, infraestructural, entre otros.

En Estrin et al. (2007) se analiza la influencia institucional en el emprendimiento por medio de una perspectiva comparativa. Los autores concluyen que la escasa actividad emprendedora es consecuencia del entorno institucional, en donde los negocios establecidos son más favorecidos por las normas y leyes, en comparación con los entrantes y sus mecanismos de impulso y desarrollo.

En Nystrom (2008) se investiga la relación entre el marco institucional, en términos de libertad económica, y el emprendimiento de autoempleo. El concepto de libertad económica contempla el tamaño del gobierno, estructura jurídica y la seguridad de los derechos de propiedad. A través de un estudio de datos de panel, se concluye que los gobiernos más pequeños, una mejor estructura legal y la seguridad de los derechos de propiedad, incrementan la capacidad emprendedora.

Friedman (2011) también relaciona la gobernabilidad con la iniciativa empresarial, en donde se supone una contribución al emprendimiento de los países, ligado a la confianza del emprendedor en el sistema gubernamental e institucional y calidad de los servicios públicos; es decir, se espera una relación positiva entre ambos. Sin embargo, sus resultados muestran un efecto negativo y significativo entre la efectividad del gobierno y la emprendedora, explicado a través de la carga fiscal, en donde las naciones con elevados índices de gobernabilidad resultan ser países con altos impuestos sobre la renta, ocasionando bajos incentivos por emprender y una disminución en la generación de nuevos negocios.

Así, Méndez et al. (2012) definen gobernabilidad como el comportamiento y rendimiento del gobierno que hace referencia a varios procesos históricos, culturales, sociales y políticos. De esta forma se argumenta la existencia de un vínculo entre gobernabilidad e instituciones. Por lo tanto, una administración gubernamental competente se manifestaría en una serie de instituciones justas e imparciales, estableciendo leyes y políticas con la capacidad de afectar la conducta de las empresas y de otros agentes económicos, ya sea implementando o cumpliendo con los derechos de propiedad, y modificando y elaborando normas para corregir las fallas de mercado.

La investigación de Álvarez y Urbano (2012) se enfoca en la influencia del entorno institucional en la actividad emprendedora, para naciones con ingreso bajo, medio y alto. Distinguen el entorno institucional en formal e informal, en donde algunos factores formales son los procedimientos para la creación de empresas y la formación en gestión y administración; mientras los informales contemplan la estabilidad política y modelos de referencia. Encuentran que los factores institucionales informales presentan una mayor contribución al emprendimiento en los países con ingreso bajo y medio, a diferencia de los países de ingreso alto, en donde los factores formales resultaron ser más determinantes.

Siguiendo esta línea, Valdez y Richardson (2013) elaboran un estudio sobre las dimensiones institucionales y el emprendimiento. Los resultados muestran cómo las dimensiones institucionales cultural-cognitiva y normativa influyen directamente en la actividad emprendedora de las naciones, mientras la dimensión institucional regulatoria no exhibe evidencia estadísticamente relevante con el emprendimiento. 
De manera similar, en Álvarez et al. (2014) se analiza, desde una perspectiva institucional, el impacto de las regulaciones en el emprendimiento de países desarrollados y en vías de desarrollo. Encuentran de qué manera el gasto público y la legislación empresarial influyen positivamente en la actividad emprendedora. Mientras otra variable institucional, como las regulaciones para el trabajo, presenta una relación distinta dependiendo del nivel de desarrollo de los países.

El trabajo de Urbano y Álvarez (2014) estudia la influencia de las dimensiones institucionales en la probabilidad de emprender, por ello especifican tres tipos de dimensiones institucionales: cultural-cognitiva, normativa y regulativa; a nivel individuo y no países. La dimensión culturalcognitiva consiste en cómo la sociedad comparte el conocimiento, en la dimensión normativa se destacan los límites del comportamiento social, mientras la dimensión regulativa comprende la capacidad de establecer, inspeccionar y ejecutar leyes y reglas. Empleando un análisis logístico, encuentran que las dimensiones institucionales aumentan la probabilidad de ser emprendedor.

También, García y Mora (2014) estudian el impacto de las instituciones, mediante el desempeño del sistema judicial e inseguridad en la dinámica empresarial. Los resultados muestran que una mayor eficacia en el sistema judicial incrementa la tasa de entrada de las empresas. Sin embargo, la eficacia judicial no mostró ningún efecto en la tasa de salida de las empresas.

En el trabajo de Avnimelech et al. (2014), se analiza la relación entre emprendimiento y la corrupción. Sus resultados muestran un efecto negativo entre la corrupción y la actividad emprendedora. Asimismo, este efecto en los países desarrollados es mayor en contraste con las naciones en vías de desarrollo. Similarmente, Mohammadi (2017) estudia el efecto entre la corrupción, calidad institucional y el emprendimiento, a través de un análisis de regresión múltiple. Su hallazgo exhibe una relación negativa de la corrupción y la iniciativa emprendedora, por otra parte, la calidad institucional muestra una relación directa con el emprendimiento.

Samadi (2019) analiza el problema de causalidad para países clasificados en diversas etapas de desarrollo. El autor encuentra matices diferenciados entre estas etapas y los efectos-causas del emprendimiento y el ámbito institucional. Por ejemplo, para las naciones en etapa iniciales, encuentra que el emprendimiento es causa del ámbito institucional. En las economías intermedias de desarrollo, la causalidad fluye del emprendimiento al elemento institucional en el corto plazo, pero en sentido contrario en el largo plazo. Mientras las instituciones de los países en etapas avanzadas, se presentan una causalidad bidireccional.

\section{METODOLOGÍA Y DATOS}

Para el desarrollo empírico de esta investigación se implementa una metodología de corte transversal, a través de Mínimos Cuadrados Ordinarios, con base en información disponible del ámbito público institucional y de emprendimiento en México, para el año 2016 y 2017, respectivamente. Su estructura es la siguiente:

$$
E M P_{i}=\alpha+\beta A A G_{i}+\gamma P I S_{i}+\delta C S P_{i}+\theta V C 1_{i}+\varphi V C 2_{i}+\varepsilon_{i} ; \quad \varepsilon_{i} \sim N\left(0, \sigma^{2}\right)
$$

Donde la variable dependiente EMP se asocia a la actividad emprendedora anual en la economía mexicana, cuyos datos se obtuvieron a partir de la Encuesta Nacional de Ocupación y Empleo (ENOE), levantada y publicada por el Instituto Nacional de Estadística y Geografía (INEGI). Esta fuente reporta el número de trabajadores independientes, definido como aquella 
persona dueña de un negocio, que trabaja en ella y/o emplea a otras personas. Por consiguiente, para esta investigación el trabajador independiente se considera como emprendedor si ha iniciado su negocio durante el año 2017. Así, con base en Texis et al. (2016), se genera una tasa de emprendimiento, la cual describe la capacidad emprendedora de cada entidad federativa de México. La tasa se calcula a partir de la siguiente fórmula:

$$
E M P_{i}=\frac{\text { Nuevos trabajadores independientes }_{2017, i}}{\text { Total de trabajadores independientes }_{2017, i}}
$$

\section{$E M P=$ Tasa de actividad emprendedora \\ $i=$ Entidad federativa}

Cabe señalar, el dividendo de la ecuación 2 sólo contabiliza aquellos trabajadores independientes que surgieron durante el año 2017. Por otra parte, el divisor incluye todos los trabajadores independientes del año 2017, este último incluye a los trabajadores independientes que iniciaron en años anteriores y siguen siendo trabajadores independientes en el 2017. La ENOE presenta registros desde el año 2005. De esta forma, la tasa de emprendimiento presenta un intervalo de valores $[0,1]$, en donde un número mayor hace referencia a una entidad federativa muy emprendedora, mientras un valor cercano a cero indica que el estado es poco emprendedor.

Para las variables independientes, se consideran indicadores institucionales del sector público, además de algunas de control, con efecto de contextualizar el análisis. Para las institucionales, los datos se extrajeron de la Encuesta Nacional de Calidad Regulatoria e Impacto Gubernamental en Empresas (ENCRIGE) del INEGI, levantada únicamente en el año 2016. Así, se consideraron aquellos indicadores que fueron de mayor interés y participación para la sociedad mexicana, las cuales son: acceso y apertura gubernamental (AAG), percepción de la inseguridad (PIS) y la calidad de los servicios públicos (CSP).

El acceso y apertura gubernamental hace referencia a la interacción por medio del internet entre el gobierno y las empresas, cuya intención es agilizar trámites burocráticos, al mismo tiempo de facilitar el acceso a la información pública; en este sentido, el indicador considera el porcentaje de empresas que han realizado al menos un trámite por internet; se espera que una mayor cobertura digital de la gestión gubernamental ocasione un aumento en la actividad emprendedora. Con respecto a la inseguridad, todo esfuerzo público en esta materia debe reflejarse en la reducción de los índices de violencia o en la percepción de inseguridad. Así, este indicador captura el porcentaje de empresas que se relacionan problemas de esta condición; se espera que un incremento en los índices de percepción de violencia o delincuencia en la entidad disminuya el emprendimiento.

La calidad de los servicios públicos mide de manera aproximada el porcentaje de empresas complacidas con las condiciones de las ciudades, específicamente en calles y avenidas; en consecuencia, una mayor satisfacción en la calidad de estos servicios públicos debe incentivar el número de emprendimientos.

El desfase de un año entre los datos de la variable dependiente sobre las independientes, coadyuva para controlar el periodo de duración del proceso del emprendimiento, en donde algunas evidencias empíricas para México han demostrado una causalidad en este sentido y, además, una duración aproximada de tres trimestres para consolidar el inicio de un nuevo negocio (González et al., 2018). De esta forma, es posible afirmar, con seguridad, que se estudia la contribución de los 
elementos institucionales del año 2016 en el fenómeno emprendedor del 2017. Esto evita que se manifiesten distintos escenarios de causalidad entre las variables disponibles, como los mencionados por Samadi (2019).

Respecto a las variables de control, se consideran el salario (VC1) y la educación (VC2); elementos ampliamente discutidos en literatura económica contemporánea como factores determinantes en el dinamismo del emprendimiento (Mungaray et al., 2016). Para efectos de facilitar la interpretación estadística de los coeficientes, se utilizan logaritmos en ambas variables. Los datos del salario se obtuvieron a través de la Secretaria de Trabajo y Previsión Social (STPS), la cual reporta el promedio estatal del salario diario asociado a trabajadores asegurados en el Instituto Mexicano del Seguro Social (IMSS) del año 2016; por lo que refleja las condiciones del salario proveniente de empleos formales. La serie de educación se extrajo del INEGI, en donde se registra el promedio de años de escolaridad formal de la población con 15 años o más, es decir, de la población económicamente activa.

Además, el intercepto se representa por $\alpha$ y el residual estadístico con el coeficiente $\varepsilon$. El prefijo $i$ especifica las entidades federativas mexicanas, es decir, el modelo agrupa datos de cada variable para todos los estados del país. Se desarrollan, conjuntamente, las pruebas de normalidad y heterocedasticidad para analizar la robustez de los resultados. En la primera se utiliza el estadístico de Jarque-Bera y en la segunda la prueba de Breusch-Pagan-Godfrey.

\section{RESULTADOS}

\subsection{Análisis descriptivo}

Antes de presentar los resultados de la estimación del modelo econométrico (1), se analizan tres gráficos (figura 1, 2 y 3), los cuales muestran la relación entre el aspecto institucional y la iniciativa emprendedora de cada una de las 32 entidades federativas en México. Cada uno de los gráficos se conforma por cuatro cuadrantes, estos se construyen a partir de la intercepción de los ejes de emprendimiento y del elemento institucional, es decir, el cruce de los ejes ocurre en donde se presenta el promedio grupal para cada variable analizada. En consecuencia, el cuadro superior derecho contiene a las entidades con mayor nivel emprendedora e institucional. El cuadrante superior izquierdo exhibe a las entidades con un menor índice institucional, pero con una actividad emprendedora superior al promedio. En el cuadrante inferior derecho abarca entidades con un elevado índice institucional y un bajo nivel emprendedor. Mientras el cuadro inferior izquierdo incluye estados con valores menores a la media respecto a emprendimiento y calidad institucional.

El primer grafico (figura 1) relaciona a la variable acceso y apertura gubernamental con la actividad emprendedora. En este se observa que tres estados del centro-occidente (Aguascalientes, Colima, Jalisco), uno del sur (Quintana Roo), pero sobre todo las entidades de la franja fronteriza norte (Baja California, Baja California Sur, Coahuila, Chihuahua, Nuevo León, Sonora y Tamaulipas), presentan un elevado nivel de acceso y apertura gubernamental y al mismo tiempo generan una alta actividad emprendedora. Es decir, ambas variables presentan relación positiva. Cabe resaltar que son entidades federativas que en los últimos años han presentado un crecimiento económico por arriba del promedio nacional. En contraparte, los estados del sur-sureste del país (Campeche, Chiapas, Guerreo, Hidalgo, Tabasco, Oaxaca y Yucatán) así como del centro (Estado de México, Michoacán, Puebla, Querétaro, San Luis Potosí), exhiben un índice de emprendimiento y de acceso y apertura gubernamental menores a las medias de la muestra, manteniendo la misma relación lógica que el primer grupo de estados, pero en condiciones adversas. 
Cabe destacar que sólo es un pequeño número de entidades los que se ubican en cuadrantes donde la relación entre las variables analizadas es negativa. Es decir, donde hay emprendimiento mayor (inferior) a la media y un nivel de acceso y apertura gubernamental inferior a la media (superior). Los estados de Durango, Guanajuato y Sinaloa muestran una iniciativa emprendedora mayor a la media, aunque con índice de acceso y apertura gubernamental menor al promedio grupal. En un sentido opuesto están la Ciudad de México, Morelos, Nayarit, Tlaxcala y Zacatecas. En consecuencia, la mayoría de los estados presentan una relación directa entre el emprendimiento y el acceso a la apertura gubernamental.

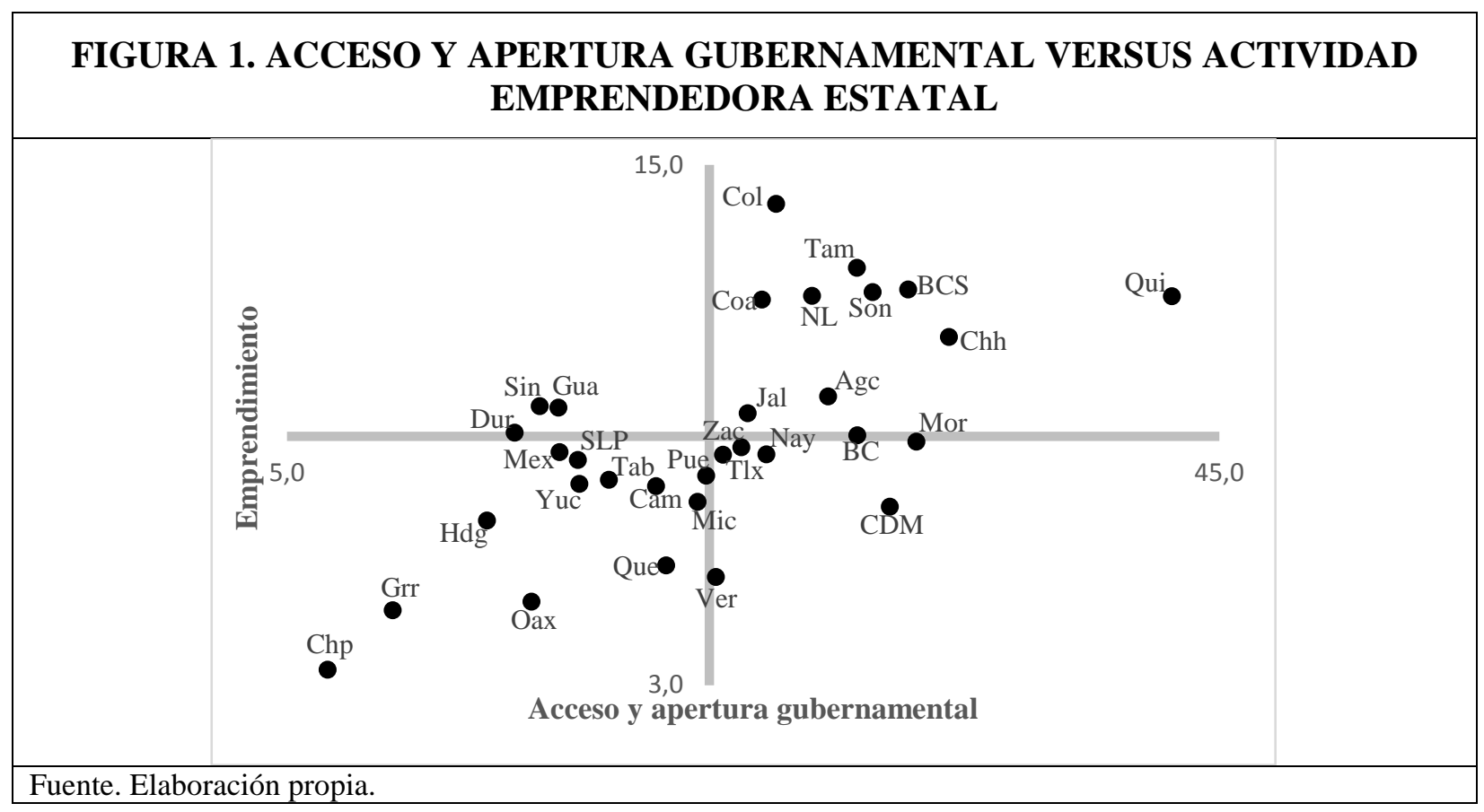

La figura 2 presenta la relación entre la actividad emprendedora y la percepción de la inseguridad de los estados mexicanos. El gráfico hace evidente la débil relación (directa o indirecta) que existe entre estas variables de estudio, resultando una distribución de estados difusa y sin ningún patrón sistemático observable a simple vista. Lo anterior puede explicarse debido a que la variable institucional es subjetiva, es decir, la percepción es un indicador de opinión, por lo que presenta una fluctuación elevada, lo que impide determinar una relación específica con la actividad emprendedora.

No obstante, realizando un esfuerzo de clasificación, se puede visualizar que los estados principales del norte y occidente del país (Chihuahua, Guanajuato, Jalisco, Nuevo León, Sinaloa, Sonora y Tamaulipas) aparecen dentro del primer cuadrante, lo que significa que estas entidades tienen una actividad emprendedora superior al promedio, a pesar de contar con una alta percepción de inseguridad. De manera similar, las entidades del sur como Campeche, Chiapas y Yucatán, además de Nayarit, Hidalgo y Querétaro, mostraron bajo emprendimiento con menores índices de percepción de la inseguridad, lo que supondría que esta condición de mínimo emprendimiento obedece a circunstancias diferentes. De acuerdo con lo esperado, el grupo de estados con alta iniciativa emprendedora y menor inseguridad comparativa está conformado por Aguascalientes, Baja California, Baja California Sur, Coahuila, Colima, Durango y Quintana Roo, cuadrante que 
reflejaría la relación ideal entre estas variables. Con la misma lógica, pero en sentido adverso, se encuentran Ciudad México, Guerrero, Estado de México, Morelos, Oaxaca, Puebla, San Luis Potosí, Tabasco, Veracruz y Zacatecas, quienes ostentaron una alta percepción de la inseguridad y una baja tasa de emprendimiento.

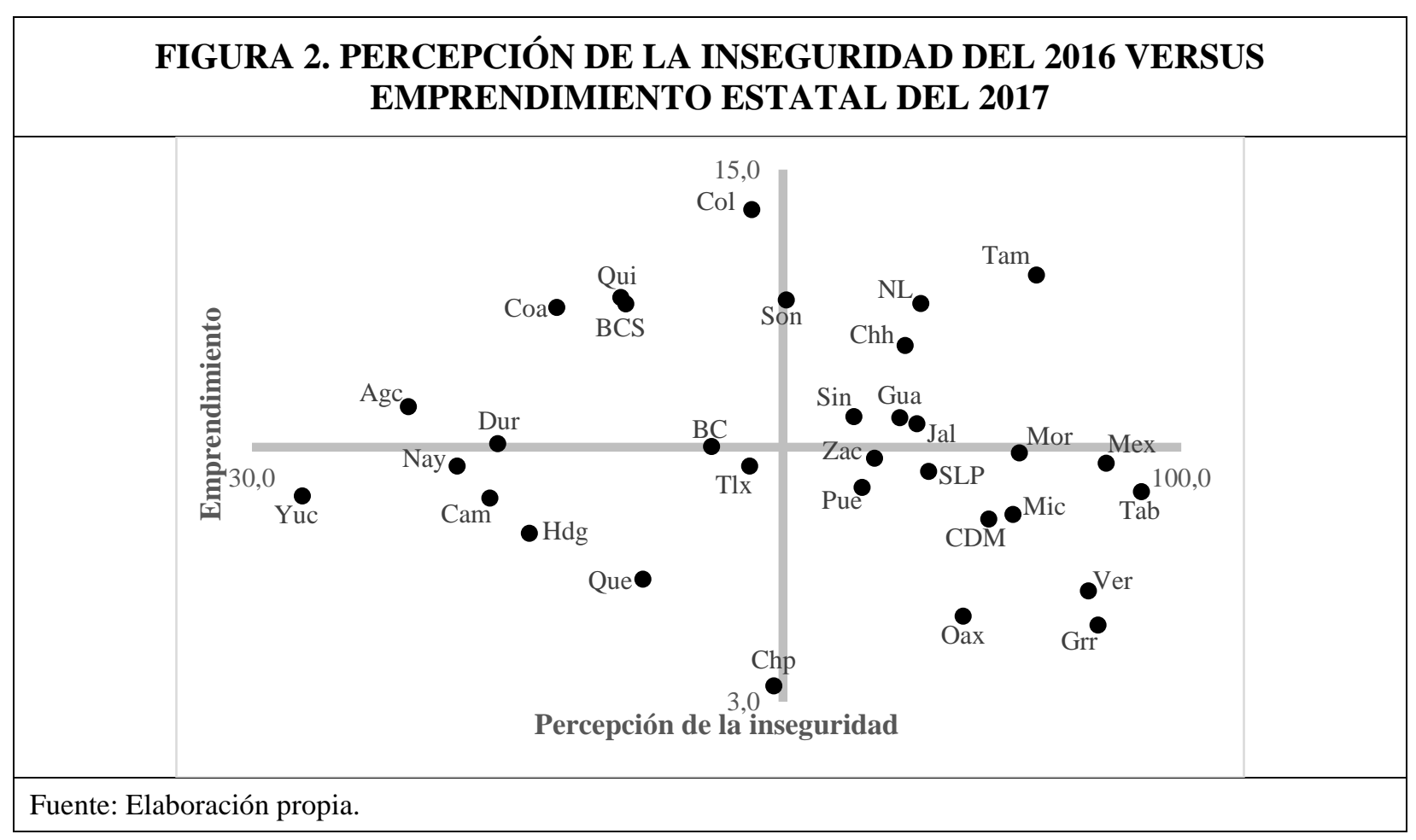

$\mathrm{Al}$ analizar la figura 3, la cual exhibe el vínculo entre la iniciativa empresarial y la calidad de los servicios públicos, es posible apreciar una ligera relación positiva. De tal forma que una mejor calidad de los servicios públicos parece indicar una mayor generación de emprendedores, este hallazgo corresponde con los estados de Aguascalientes, Baja California Sur, Colima, Durango, Guanajuato, Morelos, Nuevo León, Quintana Roo, Sinaloa y Tamaulipas. Asimismo, una menor calidad de los servicios públicos señala una baja actividad emprendedora, esto concuerda con las entidades de Campeche, Chiapas, Ciudad de México, Guerreo, Hidalgo, Estado de México, Michoacán, Oaxaca, Puebla, Tabasco, Veracruz y Zacatecas. Mientras Chihuahua, Jalisco y Sonora presentaron una alta iniciativa empresarial, pero con un índice institucional inferior a la media. Similarmente, los estados de Morelos, Nayarit, Querétaro, San Luis Potosí y Yucatán mostraron un bajo nivel de emprendimiento con respecto al promedio, pero con una alta calidad de los servicios públicos. 


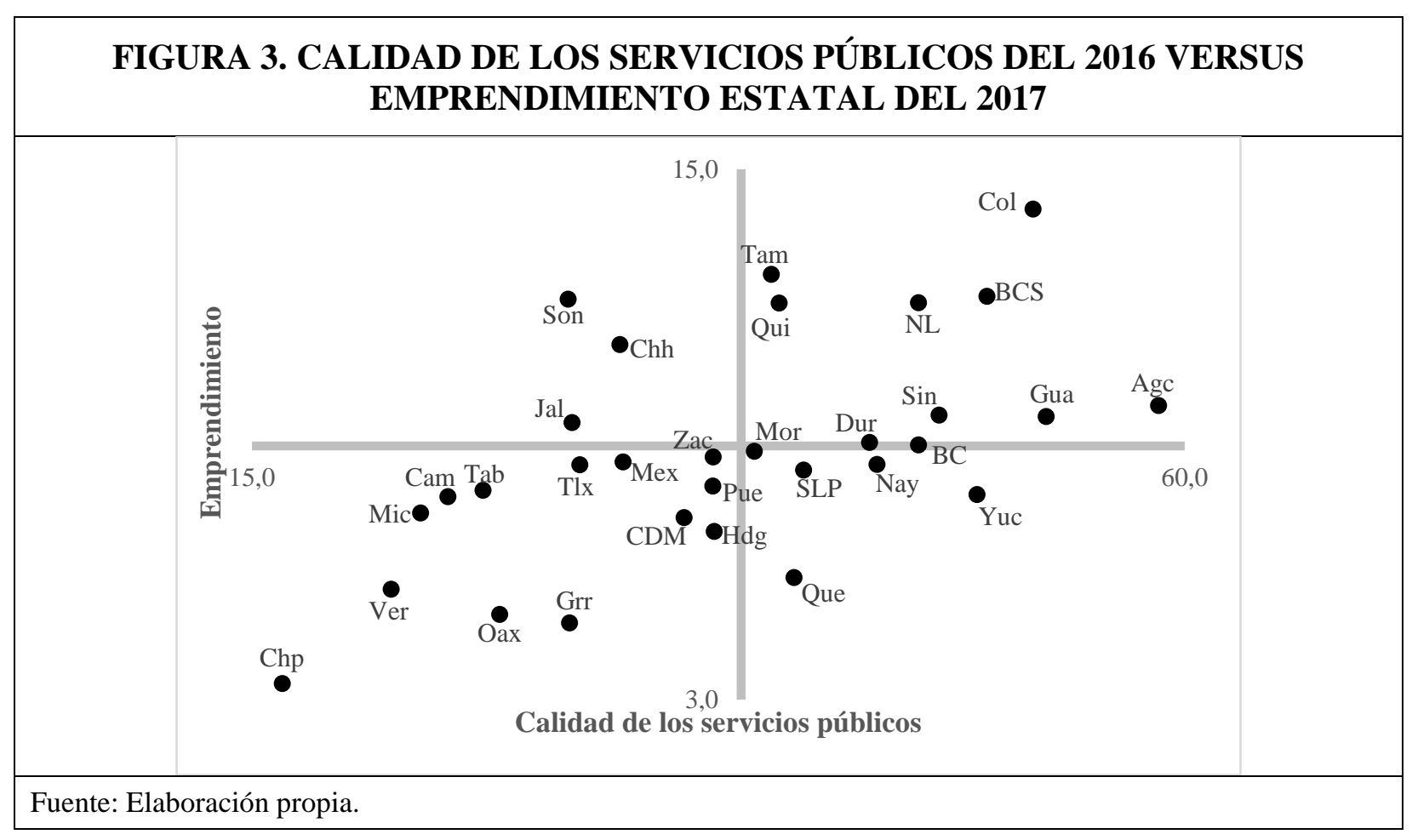

Con base en datos de la ENOE y del último Censo Económico disponible por el INEGI, se logra identificar que el 95 por cien del total de unidades productivas en México, son de nivel micro, sector en donde se ubican principalmente los emprendimientos por necesidad. No obstante, es este grupo de microempresas las que congregan más del 50 por cien del total de empleados del país.

\subsection{Hallazgos econométricos}

El cuadro 2 muestra los resultados de la estimación del modelo econométrico, lo que brinda significancias estadísticas de las variables de análisis y magnitudes de impacto. En primera instancia, el coeficiente de la variable acceso y apertura gubernamental es positivo y estadísticamente significativo al 99 por cien. Por lo tanto, se puede sugerir que un incremento en una unidad porcentual del indicador acceso y apertura gubernamental, aumenta la actividad emprendedora en 0.1333 por cien. 


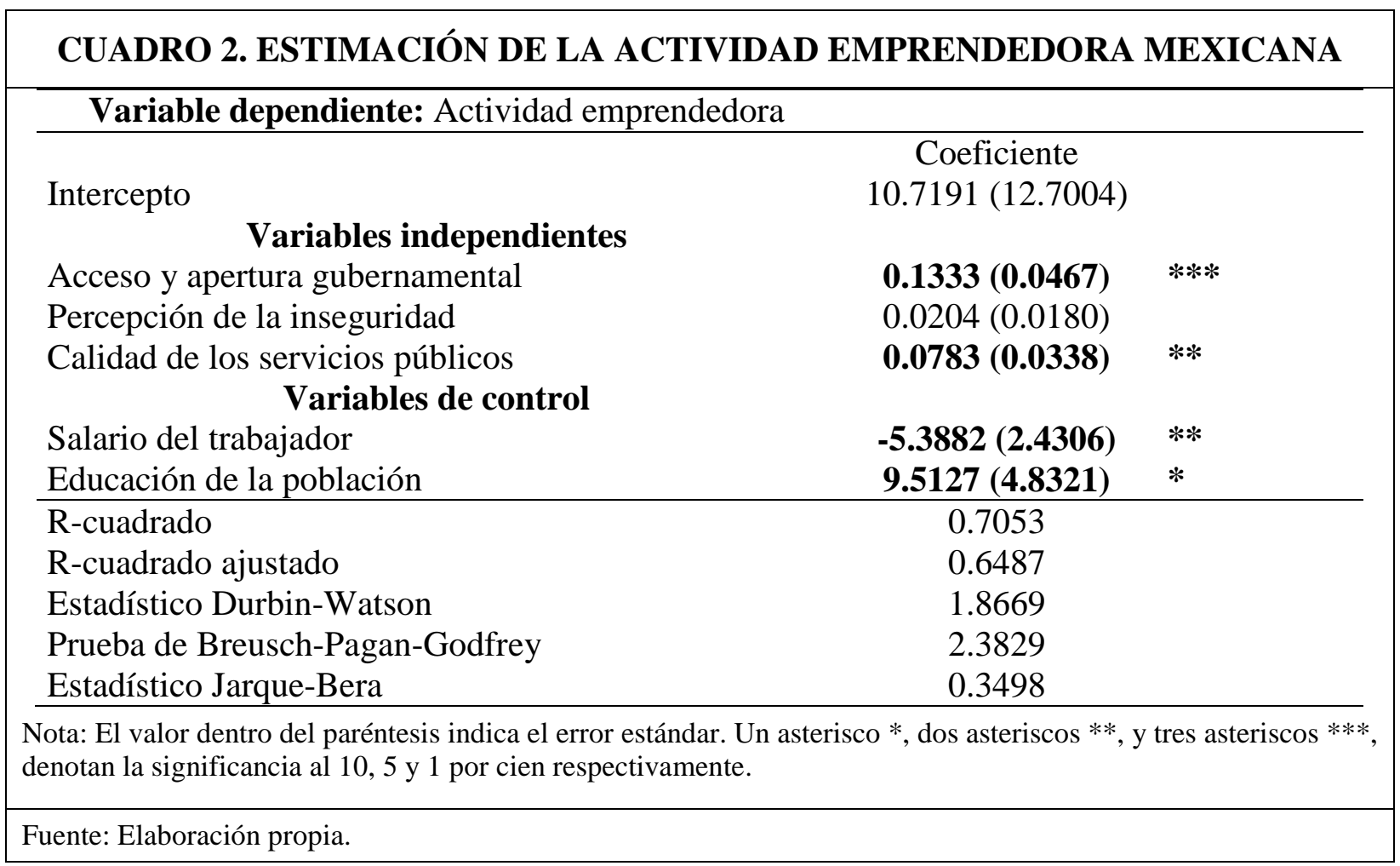

La variable percepción de inseguridad no resulta estadísticamente significativa para la actividad emprendedora, lo que comprueba la observación realizada en el apartado de análisis descriptivo. La variable institucional calidad de los servicios públicos presenta un coeficiente de 0.0783 y resultó estadísticamente significativo al 95 por cien. De esta forma, un aumento del 1 por cien en este indicador genera un incremento en el emprendimiento del 0.08 por cien.

Respecto a las variables de control, el salario del trabajador exhibe un coeficiente negativo y estadísticamente significativo al 95 por cien. El coeficiente arrojado supone que el emprendimiento disminuye en 0.05 por cien cuando el salario del trabajador aumenta en 1 por cien. Esto da pauta para suponer la comprobación adyacente del tipo de emprendimiento, en el sentido que la precarización laboral, reflejado en la disminución de las condiciones de empleo, y sobre todo, de los niveles salariales, impulsa el dinamismo del emprendimiento catalogado como de necesidad. Así, el resultado del modelo asociado a este coeficiente puede reinterpretarse que el emprendimiento podría incrementarse en 0.05 por cien cuando el salario del trabajador disminuye en 1 por cien.

Por su parte, la variable de la educación también resulta significativa, pero se manifiesta con signo contrario al salario. Es decir, el nivel educativo se relaciona de manera directa con el emprendimiento. El resultado refleja que un aumento de una unidad porcentual en los años de educación, ocasiona un incremento del 0.10 por cien en la actividad emprendedora. Esto a su vez, se asocia a una de las características fundamentales del emprendimiento, teóricamente discutido, en el sentido que el aumento de las capacidades cognoscitivas, el aprendizaje y el adiestramiento, incrementa la actividad emprendedora de las economías.

Cabe destacar que las variables explicativas en el modelo interpretan de manera considerable la actividad emprendedora en su conjunto, al presentar un R-cuadrado de 0.7053, y 0.6487 en el R- 
cuadrado ajustado. Además, esta estimación no presenta problemas de autocorrelación según el estadístico Durbin-Watson que resulta de 1.8669. Adicionalmente, se realizó la prueba de heterocedasticidad de Breush-Pagan-Godfrey, cuya hipótesis nula estipula homocedasticidad de los residuos. En la estimación del modelo no se rechaza la hipótesis nula. Para determinar si los residuos del modelo se comportan de forma normal, se implementó el estadístico Jarque-Bera, el cual no rechaza la hipótesis nula de normalidad en ningún nivel de significancia convencional.

Para sintetizar el análisis empírico, el indicador institucional de acceso y apertura gubernamental se distingue por presentar la mayor contribución a la actividad emprendedora regional en México, respecto de las variables analizadas. Mientras la variable institucional calidad de los servicios públicos reporta un coeficiente en términos absolutos mayor al salario, pero menor a la educación. Por otra parte, llama la atención como la percepción de la inseguridad no es un factor sistemáticamente considerable al momento de emprender, aunque podría ser factor determinante a nivel marginal, para cada emprendimiento en particular.

\section{CONCLUSIONES}

Desde la evidencia empírica de la diversa literatura, se robustece la idea de que la alta calidad de los servicios ofrecidos por parte del gobierno funge como parámetro respecto al buen trabajo de las instituciones. Aquí se establece que, siempre que las instituciones sean eficientes, la actividad emprendedora se verá favorecida. El marco institucional es un factor importante para determinar el dinamismo del emprendimiento, complementario y/o igual de prioritario al marco netamente económico de las regiones. Esto abona el debate teórico en el sentido de que un eficiente marco institucional es necesario para el crecimiento y desarrollo económico.

Esta investigación ratifica el papel que el gobierno debe desempeñar como pilar en el apoyo a las empresas emergentes, el cual debe ser eficaz desde cualquier punto de vista. Si bien es cierto que existen múltiples programas que van desde los apoyos financieros hasta las capacitaciones, es de igual o mayor importancia la cercanía y la facilidad de interacción que debe de existir entre los emprendedores y el ente gubernamental. Por lo anterior, resulta trascendente la figura del gobierno electrónico, digital o abierto (como algunos enfoques establecen) como una herramienta capaz de facilitar esta interacción entre el gobierno y los emprendedores, lo cual se traduce en un factor que incrementa la eficiencia institucional.

El tema de la inseguridad se aborda desde el ámbito perceptivo, es decir, subjetivo; y a pesar de no establecer una relación significativa, se deja sobre la mesa para futuras investigaciones el caso de los estados que mayor grado de emprendimiento generan, pero que a su vez son las regiones en donde se perciben los niveles más altos de inseguridad en el país.

Se puede argumentar que a pesar de que el emprendimiento es mayoritariamente gracias al propio emprendedor y su capacidad de sobreponerse ante las adversidades derivadas de factores internos (como un bajo desconocimiento de temas financieros o de mercado) y/o factores externos (como periodos de inestabilidad social y económica), es importante que el impacto institucional ayude a mitigar estos efectos y sea un soporte para la consolidación exitosa de dichos emprendimientos.

Resulta pertinente que futuras investigaciones cuestionen el sentido de causalidad entre las variables institucionales y de emprendimiento, ante diversos contextos regionales y nacionales. Debido a la estructura de los datos disponibles para esta investigación, y con base en evidencia empírica recabada y expuesta, aquí se supone un sólo sentido de causalidad, no obstante, el sentido bidireccional podría explicar ciertos dinamismos económicos, ante diversos periodos de estudio. 
En suma, la presente investigación pone de manifiesto la necesidad de generar complementariedades entre la actividad emprendedora y el entramado institucional en México, pues esto se traduciría invariablemente en mejores niveles bienestar económico. Por ello, es importante destacar que es objetivo de todos, pero principalmente de los hacedores de política pública, tomar consciencia de que las instituciones se deben conducir con eficiencia, si es que se quieren lograr resultados positivos en el emprendimiento.

\section{REFERENCIAS}

Acs, Z. (2006): “How is Entrepreneurship Good for Economic Growth?”, Innovations: technology, Governance and Globalization, vol. 1, $\mathrm{n}^{\mathrm{o}}$ 1, pp. 97-107. doi: https://doi.org/10.1162/itgg.2006.1.1.97

Acs, Z., Desai, S. y Hessels, J. (2008): “Entrepreneurship, Economic Development and Institutions”, Small Business Economics, vol. 31, n 3, pp. 219-234.

Acs, Z., Estrin, S., Mickiewicz, T., y Szerb, L. (2018): "Entrepreneurship, Institutional Economics and Economic Growth: An Ecosystem Perspective”. Small Business Economics, vol. 51, $\mathrm{n}^{\circ}$ 2, pp. 501-5014. doi: https://doi.org/10.1007/s11187-018-0013-9

Acs, Z. y Vargas, A. (2005): "Entrepreneurship, Agglomeration y Technological Change”, Small Business Economics, vol. 24, n 3, pp. 323-334. doi: https://doi.org/10.1007/s11187-0051998-4

Álvarez, C., Amorós, J. y Urbano, D. (2014): "Regulations and Entrepreneurship: Evidence from Developed and Developing Countries”, Innovar, 24, edición especial, pp. 81-89. doi: https://doi.org/10.15446/innovar.v24n1spe.47548

Álvarez, C. y Urbano, D. (2012): "Factores del Entorno y Creación de Empresas: un Análisis Institucional2, Revista Venezolana de Gerencia, vol .17, n 57, pp. 9-38.

Amorós, E. y Cristi, O. (2011): "Poverty and Entrepreneurship in Developing Countries", The Dinamics of Entrepreneurship, 209-230. doi: https://doi.org/10.1093/acprof:oso/9780199580866.003.0010

Aparicio, S., Urbano, D. y Audrestch, D. (2015): "Institutional Factors, Opportunity Entrepreneurship and Economic Growth: Panel Data Evidence”, Technological Forecasting and Social Change, $\mathrm{n}^{\mathrm{o}}$ 102, pp. 45-61. doi: https://doi.org/10.1016/j.techfore.2015.04.006

Avnimelech, G., Zelekha, Y. y Sharabi, E. (2014): "The Effect of Corruption on Entrepreneurship in Developed vs non-Developed Countries”, International Journal of Entrepreneurship Behavior and Research, vol. 20, nº 3, pp. 237-262. doi: https://doi.org/10.1108/ijebr-102012-0121

Belanger, F. y Carter, L. (2008): “Trust and risk in E-government adoption”, The Journal of Strategic Information System, vol. 17, $\mathrm{n}^{\mathrm{o}}$ 2, pp. 165-176. doi: https://doi.org/10.1016/j.jsis.2007.12.002

BID, Banco Interamericano de Desarrollo (2011): Strengthening Transparency and Accountability for Improved Governance, Ed. BID, Washington, D.C.

Bochma, N., Content J., Sanders M. y Stam, E. (2018): "Institutions, Entrepreneurship, and Economic Growth in Europe”, Small Business Economics, pp. 1-17. doi: https://doi.org/10.1007/s11187-018-0012-X

Chingos, M. (2012): “Citizen Perception of Government Service Quality: Evidence from Public Schools”, Quarterly Journal of Political Science, vol. 7, $\mathrm{n}^{\circ}$ 4, pp. 411-445. doi: https://doi.org/10.1561/100.00011071 
Davari, A. y Farokhmanesh, T. (2017): “Impact of Entrepreneurship Policies on Opportunity to Startup”, Management Science Letters, $\mathrm{n}^{\mathrm{0}}$ 7, pp. 431-438. doi: https://doi.org/10.5267/j.msl.2017.6.003

De Wit, G. y De Kok, J. (2013): "Do Small Business Create More Jobs? New Evidence for Europe”, Small Business Economics, vol. 42, $\mathrm{n}^{\mathrm{o}}$ 2, pp. 283-295. doi: https://doi.org/10.1007/s11187-013-9480-1

Desai, M., Gompers, P. y Lerner, J. (2003): Institutions, Capital Constraints, and Entrepreneurial Firms Dynamics: Evidence from Europe, National Bureau of Economic Research, Working Papers Series 10165. doi: https://doi.org/10.3386/w10165

Estrin, S., Aidis, R. y Mickiewicz, T. (2007): “Institutions and Entrepreneurship Development in Russia: A Comparative Perspective2, Journal of Business Venturing, no 23, pp. 656-672. doi: https://doi.org/10.2139/ssrn.1017252

Fairlie, R. y Fossen, F. (2017): Opportunity versus Necessity Entrepreneurship: Two Components of Business Creation, SIERPR Discussion, doi: https://doi.org/10.2139/ssrn.3010267

Friedman, B. (2011): "The Relationship between Governance Effectiveness and Entrepreneurship”, International Journal of Humanities and Social Science, vol. 1, $\mathrm{n}^{\mathrm{0}}$ 17, pp. 221-225.

García-Posada, M. y Mora-Sanguinetti, J. (2014): Entrepreneurship and Enforcement Institutions: Disaggregated Evidence for Spain. Banco de España, Documento de Trabajo 1405. Madrid. doi: https://doi.org/10.2139/ssrn.2413422

González, J. (2009): Teoría del desarrollo económico neoinstitucional, una alternativa a la pobreza del siglo XXI, Primera edición. Universidad de Colima. H. Cámara de Diputados, LX Legislatura, Porrúa. México.

González, J., Osorio, G. y Mungaray, A. (2018): "La microempresa mexicana, un asunto de necesidad y no de oportunidad: el caso de Colima”, Revista Análisis Económico, vol. 33, nº 84, pp. 123-142.

Horsburgh, S., Goldfinch, S. y Gauld, R. (2011): "Is public trust un government associated with trust in E-government?”, Social Science Computer Review, vol. 29, n 2, pp. 232-241. doi: https://doi.org/10.1177/0894439310368130

Kantis, H. (2008): Emprendedores de Origen Humilde: ¿Cómo Incide la Estructura Social en la Creación de Empresas en América Latina?, BID y FUNDES Internacional, Santiago.

Kantis, H., Angelelli, P. y Koenig, V. (2004): Desarrollo Emprendedor: América Latina y la Experiencia Internacional. BID y FUNDES Internacional, Santiago.

Luksha, P. (2008): "Niche Construction: The Process of Opportunity Creation in the Environment", Strategic Entrepreneurship Journal, vol. 2, $\mathrm{n}^{\mathrm{0}}$ 4, pp. 269-283. doi: https://doi.org/10.1002/sej.57

Méndez-Picazo, M., Galindo-Martín, M. y Ribeiro-Soriano, D. (2012): “Governance, Entrepreneurship and Economic Growth”, Journal of Entrepreneurship and Regional Development, vol. $\quad 24, \quad \mathrm{n}^{\circ} \quad 10, \quad$ pp. 865-877. https://doi.org/10.1080/08985626.2012.742323

Mohammadi, M. (2017). “Institutions and entrepreneurship: the mediating role of corruption”, World Journal of Entrepreneruship, Management and Sustainable Development, vol. 13, ${ }^{\circ}$ 3, pp. 262-282. doi: https://doi.org/10.1108/wjemsd-09-2016-0045

Mungaray, A., Aguilar, J. G. y Osorio, G. (2016): "Rethinking the purpose of micro-enterprises in developing countries: Evidence for Mexico”, Journal of Developmental Entrepreneurship, vol. 21, n 3, pp 1-16. doi: https://doi.org/10.1142/S1084946716500199 
Mungaray, A., Osorio, G. y Ramírez, N. (2017): Ensayos económicos sobre microempresas de subsistencia en México, MA Porrua-UABC, México.

Munagaray, A., Ramírez, N. y Aguilar, J. G. (2016): Economía del emprendimiento y las pequeñas empresas en México, MA Porrua-UABC, México.

North, D. (1993): Instituciones, Cambio Institucional y Desempeño Económico, Fondo de Cultura Económica, México.

Nystrom, K. (2008): “The Institutions of Economic Freedom and Entrepreneurship: Evidence from Panel Data”, Springer, vol. 136, n 3-4, pp. 269-282. doi: https://doi.org/10.1007/s11127008-9295-9

Plummer, L., Haynie, J. y Godesiabois, J. (2007): “An Essay on the Origins of Entrepreneurial Opportunity”, Small Business Economics, vol. 27, $\mathrm{n}^{\mathrm{o}}$ 4, pp. 363-379. doi: https://doi.org/10.1007/s11187-006-9036-8

Saavedra, R. y Texis, M. (2018). El factor institucional en el emprendimiento por oportunidad de América Latina y el Caribe, working paper.

Samadi, A. (2019). Institutions and entrepreneurship: unidirectional or bidirectional causality?. Journal of Global Entrepreneurship Research, vol. 9, $\mathrm{n}^{\mathrm{o}}$ 1. doi: https://doi.org/10.1186/s40497-018-0129-z

Shane, S. y Venkataraman, S. (2000): “The Promise of Entrepreneurship as a Field of Research”, Academy of Management Review, vol. 25, nº 1, pp. 217-226. doi: https://doi.org/10.1007/3540-48543-0_8

Swianiewicz, P. (2001). Between Active Appreciation, Passive Approval and Distrustful Withdrawal, Citizens' Perception of Local Government Reforms and Local Democracy in Central-Eastern Europe. working paper, Budapest, Hungría.

Tapia, M. (2010): Organizaciones de la Sociedad Civil y Políticas Públicas”, edición los Grandes Problemas de México, Ed. El Colegio de México, México.

Texis, M., Saavedra, R. y Aguilar, J. (2016). El papel del capital emprendedor en México, 20002014. MA Porrua-UABC, México.

Tolbert, C. y Mossberger, K. (2006): "The effects of E-Government on trust and confidence in Government”, Public Administration Review, vol. 66, $\mathrm{n}^{0}$ 3, pp. 354-369. doi: https://doi.org/10.1111/j.1540-6210.2006.00594.x

Urbano, D., y Álvarez, C. (2014): "Institutional Dimensions and Entrepreneurial Activity: An International Study”, Small Business Economics, vol. 42, $\mathrm{n}^{\circ}$ 4, pp. 703-7016. doi: https://doi.org/10.1007/s11187-013-9523-7

Valdez, M. y Richardson, J. (2013): “Institutional Determinants of Macro-Level Entrepreneurship”, Entrepreneurship Theory and Practice, vol. 37, nº 5, pp. 1149-1175. doi: https://doi.org/10.1111/etap.12000

Van de Walle, S. y Geert, B. (2003): "Public Service Performance and Trust in Government: The Problem of Causality”, International Journal of Public Administration, vol. 26, nº 8-9, pp. 891-913. doi: https://doi.org/10.1081/pad-120019352 